\title{
Communiquer et agir en commun : le cas d'un journal interne instrumentalisé
}

Ivan Ivanov

\section{(2) OpenEdition}

1 Journals

Édition électronique

URL : http://journals.openedition.org/communicationorganisation/4231

DOI : 10.4000/communicationorganisation.4231

ISSN : $1775-3546$

Éditeur

Presses universitaires de Bordeaux

Édition imprimée

Date de publication : 1 juin 2013

Pagination : 213-226

ISBN : 978-2-86781-883-7

ISSN : $1168-5549$

Référence électronique

Ivan Ivanov, "Communiquer et agir en commun : le cas d'un journal interne instrumentalisé », Communication et organisation [En ligne], 43 | 2013, mis en ligne le 01 juin 2015, consulté le 30 avril 2019. URL : http://journals.openedition.org/communicationorganisation/4231 ; DOI : 10.4000/ communicationorganisation.4231 


\title{
Communiquer et agir en commun : le cas d'un journal interne instrumentalisé
}

\author{
Ivan Ivanov'
}

Avec quelque 40 millions de lecteurs en France $^{2}$, le journal interne connaît un véritable succès au sein des organisations ${ }^{3}$. Il est le support écrit principal de la communication interne (Détrie, Broyez, 2003). Mais malgré sa popularité, le journal interne est un objet de recherche « abandonné » par les sciences de l'information et de la communication (SIC) et par les sciences de gestion. Après l'intérêt qu'il a suscité dans les années 1980-1990, il est devenu rapidement un support de la communication interne parmi d'autres (Argenti, 2006).

Deux approches - fonctionnaliste et critique - ont donné lieu à de nombreux travaux (Agnès, Durier, 1992 ; Wild, 1986 ; Nicotri, 2001; Deley, 1995 ; Morillon, 2005, 2009; Lambert, 1993). Pour elles, le journal interne accomplit des missions officielles ou tues; il est un moyen de circulation d'information ou un outil de gestion des personnels, le rôle des derniers dans ce processus étant souvent perçu comme passif. En revanche, à notre connaissance, aucune d'entre elles n'a étudié le processus d'écriture, d'élaboration et d'utilisation du journal interne par les différents membres de l'organisation, dans un temps long, en train de se faire, en réintégrant le rôle actif de l'individu. Il manque donc d'études qui s'attachent à expliquer comment les individus s'investissent dans l'élaboration du journal interne pour organiser individuellement leur travail quotidien en accomplissant des actes collectifs.

Afin d'explorer cette problématique, nous étudions le processus d'élaboration d'un journal interne en temps réel, de l'intérieur de l'organisation qui le produit, en mettant en place une observation participante et une méthode

1 Ivan Ivanov est docteur en sciences de l'information et de la communication à l'université de Toulouse 3, le Mirail. Il travaille au sein du Laboratoire d'Études et de Recherches Appliquées en Sciences Sociales, équipe Organicom ; ivan_ivaniv@voila.fr

2 Source «Communication et entreprise », http://www.communicationetentreprise.com/.

3 Il est impossible de dénombrer aujourd'hui le nombre exact de journaux internes puisqu' " INSS France » n'attribue pas d'INSS (International Standard Serial Number) aux journaux internes. Selon l'Association Française de Communication Interne (AFCI, http://www.afci.asso.fr/publications/categorie/toutes), 85 \% des entreprises possèdent un ou plusieurs journaux internes. 
d'analyse mixte (Aldebert, Rouzies, 2011) qui vise à faire connaître les auteurs des textes et leurs stratégies personnelles et collectives. Nos résultats d'analyse montrent que le journal interne a une fonction organisante qui est initialement imprévue et inattendue par les auteurs des textes lors du lancement du journal interne.

Dans un premier temps, nous présentons les approches fonctionnaliste et critique portant sur le journal interne et nous proposons de mobiliser la communication organisationnelle comme cadre d'analyse. Ensuite, nous détaillons le positionnement du chercheur sur son terrain de recherche, ainsi que la collecte des données et leur méthode d'analyse mixte et longitudinale. Dans un troisième temps, les résultats d'analyse de 145 textes publiés (entre 2009 et 2011) depuis la création du journal interne sont précisés. Ils dévoilent comment le journal interne est instrumentalisé par les différents membres de l'organisation pour atteindre des finalités personnelles et collectives.

\section{Le journal interne organisant}

Le journal interne peut être étudié à travers deux approches traditionnelles selon lesquelles il accomplit des missions officielles ou dissimulées. Au-delà de ces deux visions, une approche organisante (Giroux, 1994) souligne que son premier objectif est d'organiser les processus humains qui se déroulent quotidiennement au sein d'une organisation à travers les interactions.

\section{Un journal polyphonique}

Les deux approches traditionnelles qui cohabitent conjointement dans les organisations fournissent des pistes pour comprendre l'élaboration du journal interne. La première - fonctionnaliste - l'appréhende comme le journal des personnels, et la deuxième, davantage critique, voit en lui la " voix de son maître ".

Pour toutes les deux, le journal interne est un support écrit de la communication interne ${ }^{4}$ : il est un moyen de circulation d'information au sein des organisations qui crée un lien entre les personnels et la direction, ce qui nécessite une certaine adhésion, compréhension, partage de l'information, mais aussi des finalités et des enjeux organisationnels communs. Ce lien se bâtit à travers la circulation de l'information montante, descendante et latérale (Carayol, 1994) qui implique les différentes strates de la hiérarchie. Dans ce processus, la participation des membres du personnel est nécessaire afin que le journal soit perçu comme crédible et proche des lecteurs. Pour que les individus s'approprient le journal et son contenu, ce dernier doit être élaboré par et pour eux et perçu comme un moyen communicationnel transparent et

4 Cette dernière est définie ici comme une communication stratégique qui implique la participation de tous les membres du personnel dans la réalisation des divers projets. Cette définition invite à considérer la communication interne comme le résultat de choix rationnels, pris dans un but spécifique et réalisés à travers les interactions. 
autonome (Agnès, Durier, 1992). Ses textes doivent permettre aux personnels de s'exprimer et s'adressent directement à eux (Wild, 1986). Cette proximité crée du lien et de la confiance entre les personnels et la direction. "Le journal d'entreprise est porteur d'un projet généreux : rapprocher les hommes et leur apprendre à mieux se connaître au sein d'une organisation, éclairer celle-ci d'une clartéfinalement démocratique " (Lardellier, 1998). Le journal interne est donc un moyen de reconnaissance (Honneth, 2000 ; 2008) et de valorisation des individus au travail qui vise à octroyer un sentiment de respect et d'estime de soi.

Mais si les personnels sont les plus souvent cités, photographiés et nommés sur les pages du journal interne, ils prennent la parole moins souvent que les cadres et la direction (Deley, 1995). En plus, les personnels non cadres ne proposent que très rarement des idées de textes à publier, même s'ils s'y expriment souvent. Et si cela se produit en réalité, ces propositions sont habituellement " coordonnées » et "suggérées ", voire contrôlées explicitement par la direction, sous l'effet de la censure et de l'autocensure. Vincent-Bernard Nicotri (2001) parle ainsi de "polyphonie contrôlée " pour exprimer l'idée que sous une apparence de pluralité des locuteurs, le journal interne articule les mêmes sources d'information et canaux de communication. Dès lors, le journal interne accomplit des missions tues; il est en réalité au service des dirigeants.

Une fois rédigés, les textes subissent de multiples corrections et validations " politiques » (Both, 2006) qui prennent beaucoup de temps et peuvent changer radicalement le sens du texte en modifiant directement les propos des personnes interviewées. Anne Both (2006) appelle ce type de corrections " filtre managérial " qui unifie les propos des interviewés : souvent les personnels disent des choses dans le journal interne qu'ils n'ont jamais dites en réalité auparavant. Or, la « véracité » des textes n'est guère requise. Le texte devient présent et acquiert une "véracité " par le fait qu'il est publié sur les pages du journal interne, lui-même validé par les managers et la direction. Le journal interne ne fait donc que donner l'impression qu'il est élaboré par et pour les personnels. Même si les personnels y prennent souvent la parole pour s'exprimer, il est en réalité produit par les individus positionnés au plus haut niveau de la hiérarchie pour atteindre des finalités stratégiques et personnelles.

\section{De l'événement subi à l'événementialité construite}

Ce qui permet de mieux connaître les finalités de ceux qui proposent les publications et ceux qui y parlent sont les événements, en lien ou non, avec des actualités organisationnelles observables dans les différents textes du journal interne. Un événement est souvent considéré comme une action-rupture qui advient dans une "réalité » et qui apporte une " vérité ». Mais « tout élément peut être considéré comme événement dans la mesure où on le considère situé dans l'irréversibilité temporelle, comme une manifestation ou une actualisation» (Morin, 1972, p. 17). Toute chose est donc marquée d'une certaine événementialité. 
Nous proposons d'appréhender les événements sur lesquels portent les textes du journal interne de deux manières générales. Tout d'abord, comme quelque chose qui s'impose à l'organisation, quelque chose d'actuel qui survient et qui l'atteint. Ces événements sont "subis » (Labasse, 2006) et ne peuvent paraître autrement que « comme accidents ». Mais il existe aussi

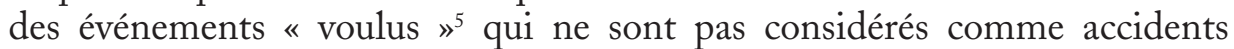
mais comme quasi-quotidiens, programmés, cycliques. Les deux types d'événements peuvent donner lieu à différentes publications dans le journal interne car ils s'appuient constamment sur le fil rouge de l'actualité.

Ces deux manières d'appréhender les événements sont pourtant très réductrices ${ }^{6}$. Nous proposons donc de parler plutôt de " provénements » (Zarifian, 1995), une notion qui permet d'appréhender les événements comme des constructions textuelles qui intègrent le rôle actif des individus dans leur construction, qui deviennent présents et commencent à exister sur les pages du journal interne. Le provénement «est quelque chose qui advient, dans le réel, quelque chose qui est en effet en rupture avec le déroulement régulier des phénomènes " (Zarifian, 1995, p. 22) et auquel les individus accordent de l'importance. Il est, dans la vie organisationnelle, quelque chose qui fait événement : un événement provoqué qui n'en reste pas moins événement. Définis ainsi, les provénements permettent d'étudier de quoi parlent les textes du journal interne, ce « quoi » étant appréhendé comme un résultat des choix personnels de ceux qui proposent et de ceux qui signent les textes.

Si les différents membres de la direction participent à l'élaboration des textes en vue d'une finalité personnelle, quel est alors le rôle du journal interne pour les organisations? Que change-t-il et que transforme-t-il dans les organisations?

\section{L'agentivité textuelle}

Au-delà des approches fonctionnaliste et critique, les travaux des chercheurs de l'École de Montréal suggèrent que certains textes, dans certaines conditions, ont la capacité d'agir et de faire agir sur l'organisation et ses membres (Taylor, Van Every, 2000), l'action étant définie ici comme «une transformation d'états opérée par un agent " (Cooren, 2013, p. 34). Ces travaux s'inscrivent dans la communication organisationnelle (Bouzon, Meyer, 2006 ; Mumby, Stohl, 1996 ; Deetz, 2008) qui a pour point de départ la façon dont les membres d'une organisation interagissent en fonction de leurs logiques divergentes et

\footnotetext{
5 Selon Pierre Labasse, il s'agit notamment des inaugurations, des journées d'études, des réunions diverses, des vœux de départs, etc.

6 Dans le cas des événements "subis ", l'existence d'un contexte extérieur s'imposant à l'organisation est supposé, alors que rien ne nous laisse dire que le contexte extérieur "impose » les événements de la même manière dans des situations différentes. Quant aux événements « voulus », ils sont tout de même «voulus » envue-et-en-lien d'un environnement (extérieur ou intérieur) et sont orientés par lui. Pourtant, il est impossible de rendre compte d'un événement sous la catégorie « effet de l'environnement » puisqu'il est immanent à la situation où il advient.
} 
des contraintes institutionnelles. Les textes du journal interne ne sont pas nés ex nibilo, mais sont construits collectivement dans l'espace et dans le temps par une multitude d'individus à travers les interactions (Mead, 1963).

L'approche textual agency (dans le sens proposé par François Cooren, 2004) conceptualise cette capacité des textes d'agir sur l'organisation - et de faire agir l'organisation sur ses membres - en créant une différence dans la structure organisationnelle. Ce qui permet de mieux comprendre l'agentivité textuelle, c'est la notion de performativité (Austin, 1962 ; Searle 1970) qui définit en quoi les énoncés écrits et oraux ne servent pas seulement à décrire les choses, mais aussi à faire des choses (Latour, 1994). Dans cette perspective, les organisations ne sont jamais des entités « données ", mais "performées » par les multiples textes, pour " une autre première fois " (Cooren, 2013), eux-mêmes produits d'une pluralité d'agentivités humaines et non humaines. L'agentivité ici n'est pas une qualité textuelle, mais un processus d'écriture (Denis, 2006) structurant pour l'organisation. Considéré à travers cette approche, le journal interne est constitué par des textes qui ne sont pas simples miroirs de la « réalité » organisationnelle et des événements qui l'atteignent, mais des agents qui créent l'organisation à travers les provénements ${ }^{7}$.

Alors, en quoi - et de quoi - la conception quotidienne du journal interne peut-elle être organisante? Quelle est plus précisément la capacité du journal interne d'agir sur l'organisation?

\section{Observer en temps réel}

Notre terrain de recherche est une organisation régionale française très hiérarchisée ${ }^{8}$ dans laquelle les personnels ont des niveaux et des statuts disparates et inégaux. Nous intégrons son service Communication en tant que communicant chargé de la rédaction des textes d'un journal interne, nommé ici Passerelle. Nous suivons à la trace le processus d'élaboration du journal interne et de ses textes et nous menons une recherche de type Grounded theory (Strauss 1992) : elle est inductive et part de l'exploration du terrain, de la collecte des faits observés et empiriques.

Après une période de deux ans de collecte des données qualitatives et quantitatives, nous procédons à une analyse mixte (Aldebert, Rouzies, 2011) et longitudinale de 11 numéros (145 textes) de Passerelle (depuis sa création).

\footnotetext{
7 C'est dans ce sens que nous pouvons affirmer que les textes « écrivent " l'organisation (Cooren, 2004), l'organisation étant appréhendée ici comme "construite en action » (" grounded in action ») (Fairhurst, \& Putnam, 2004) où textes et structure organisationnelle interagissent et se co-construisent.

8 Pour des rasions de confidentialité, son vrai nom ne sera pas divulgué.
} 


\section{Statut singulier du chercheur}

L'organisation que nous intégrons en tant que salarié chargé de rédiger les textes du journal interne, sans pour autant participer aux choix rédactionnels, est régionalement éclatée et ses multiples services et départements sont très cloisonnés. Les 839 membres du personnel sont rémunérés selon une grille institutionnelle stricte divisée en plusieurs niveaux. Les carrières y sont complexes et les promotions (attribution de niveaux supérieurs) sont rares $^{9}$. Deux parcours de carrière professionnelle y sont possibles : les parcours normés concernent les métiers typiques (appelés encore métiers-cœurs) de l'organisation où les promotions suivent une évolution réglementée, et les parcours non normés (métiers atypiques) pour lesquels il n'existe pas de règles officielles indiquant comment un membre du personnel peut passer d'un niveau à l'autre.

Cette organisation est composée de trois branches et de deux sous-directions, elles-mêmes divisées en plusieurs départements et services, qui ont des budgets inégaux pour financer divers projets, ce qui explique un fort cloisonnement interne. Un nouveau directeur arrive en 2008 et souhaite faire travailler rapidement toutes les branches et sous-directions ensemble. Il annonce la mise en place d'un projet d'entreprise et la mobilisation de la communication interne afin que cette transversalité se réalise en pratique. Sa volonté est de décloisonner les unités et de faire naître des projets interservices dans lesquels chaque unité va pouvoir connaître ce que les autres unités font tous les jours. La création et la mise en place du journal interne s'inscrivent dans ce projet stratégique de décloisonnement organisationnel. Passerelle doit donc porter des missions et des objectifs initiaux propres au projet d'entreprise afin de le faire connaître.

Nous effectuons une observation participante in situ et in vivo (Giordano, Musca, 2012) qui se caractérise par une présence prolongée sur le terrain de recherche et qui positionne le chercheur en amont, pendant et en aval des événements qui arrivent ou qui émergent à travers les interactions. L'observation participante in vivo favorise la sérendipité (Bourcier, Van Andel, 2011) qui permet de découvrir des données et des résultats auxquels le chercheur ne s'attendait pas (Sandri, 2013) susceptibles de dévoiler des aspects et des éléments nouveaux, imprévus et inexplorés jusqu'au moment de leur découverte.

Les données qualitatives (recueillies dans un journal de bord) et quantitatives colletées (textes définitifs et versions intermédiaires du journal interne) sont complétées par un recueil documentaire (33 documents écrits et électroniques internes et externes). Le journal de bord contient des observations sur des comportements, interactions, conversations, échanges formels et informels et traces physiques observées en temps réel. Les données quantitatives

9 Un système d'attribution de points est privilégié mais il ne permet pas de changer de niveau hiérarchique. 
sont constituées de 145 textes (onze numéros du journal interne) publiés et de quatre versions intermédiaires de chaque texte du journal interne (propositions, corrections, validations, justifications, annulations ou décalages de publication).

\section{Une méthode d'analyse mixte}

Les données collectées sont analysées en mobilisant une méthode mixte et longitudinale qui se veut pragmatique et qui part des questionnements définis et non pas des données collectées. Elle triangule l'analyse et l'interprétation de deux types de données : quantitatives et qualitatives.

La première grille d'analyse quantitative contient six colonnes : les quatre premières visent à coder des données portant sur les propriétés des textes étudiés, et les deux dernières sur l'identité des auteurs des textes (qui propose ? qui parle ?). Les textes sont numérotés par ordre chronologique de publication (de 1 à 145) et les titres des textes correspondants sont indiqués dans la colonne suivante. Dans une troisième colonne, les textes sont identifiés par type (témoignage ou synthèse), ce qui vise à savoir si le texte est écrit sur témoignage d'un membre du personnel ou à partir d'une synthèse de documents divers. Pour chaque type de texte - dans la colonne suivante - est associée la rubrique dans laquelle le texte est publié afin de repérer l'emplacement du texte, mais aussi de savoir si sa longueur est plus ou moins grande ${ }^{10}$. La colonne suivante (" source»), qui indique sur quelle branche ou sous-direction porte le sujet du texte, est suivie de la catégorie " auteur » qui indique précisément qui parle dans le texte.

Parallèlement, les données qualitatives sont codées dans sept grilles thématiques, une pour chaque rubrique. La colonne " numéro » permet de repérer rapidement le numéro de parution et de le reporter au sujet (catégorie "sujet») qui lui correspond. Chaque sujet publié est reporté à son numéro de parution et à sa rubrique. Ce codage trace la raison d'être de chaque texte : son message, la raison de sa proposition, réalisation et publication (motifs officiels). La troisième colonne " source/branche " (qui propose le sujet ?) identifie l'unité (branche, sous-direction...) à l'origine du sujet. Dans la catégorie suivante, "transformation ", sont indiquées les transformations observées, en lien ou non avec un événement, qui ont fait l'objet de publications sous forme d'actualité. Lidée est d'associer à chaque publication une transformation interne ou externe. Cette catégorie permet de situer la proposition d'un texte dans un moment organisationnel précis (catégorie « contexte»), tout en mettant en évidence la raison de sa parution.

Une fois les événements identifiés, les textes sont liés aux actualités du type $\mathrm{R}$ (strictement régionales), $\mathrm{N}$ (nationales : lois, décrets, etc.) ou $\mathrm{RN}$ (origine nationale qui nécessite des actions concrètes à l'intérieur de l'organisation).

10 Certaines rubriques sont plus volumineuses que d'autres, ce qui témoigne de leur importance. 
Le but de cette catégorisation est de savoir si le journal interne médiatise - ou crée - des événements, qui, du fait de leur textualisation, deviennent - ou non - des faits organisationnels signifiants qui contiennent des finalités personnelles.

Les premiers résultats obtenus grâce à la grille quantitative sont triangulés, dans une perspective longitudinale, avec l'analyse des données qualitatives codées dans sept grilles thématiques.

\section{Un journal interne instrumentalisé ?}

Nos résultats d'analyse dévoilent que le journal interne est élaboré par une multitude d'auteurs ${ }^{11}$ et coauteurs, plus ou moins bien positionnés dans la hiérarchie. Chacun d'entre eux s'approprie, instrumentalise et utilise le journal interne pour réaliser des stratégies personnelles et collectives. Bien que Passerelle n'accomplisse pas ses objectifs stratégiques initiaux imposés par le directeur et son projet d'entreprise, le journal interne a la capacité d'agir sur l'organisation et ses membres, une capacité imprévue et inattendue.

\section{Qui participe à l'élaboration du journal interne et pourquoi ?}

Les premiers résultats quantitatifs montrent qu'aucun des textes publiés n'est proposé par un membre du personnel mais par les cadres de direction (sauf sept proposés par le responsable du service Communication). Ils définissent le message et l'angle du texte, choisissent et autorisent les personnels à y participer afin d'y témoigner et signer les textes. Les cadres de direction sont les auteurs parce qu'ils ont l'autorité de faire des choix rédactionnels, mais aussi d'autoriser les témoignages des personnels. Une fois autorisés, ils témoignent avec leur propre voix, signent les textes et deviennent coauteurs.

À travers ce « jeu d'autorisation collective ", des stratégies promotionnelles sont mises en lumière : les publications de certains textes (68 témoignages), dans certaines conditions, sont suivies de promotions $(27$ personnes promues) pour ceux qui y témoignent (personnels) et, parfois, pour ceux qui les proposent (cadres de direction). Dans la totalité des textes étudiés, la personne promue exerce un métier atypique (parcours non normé) pour l'organisation : l'évolution des carrières professionnelles des métiers atypiques n'est pas prédéfinie comme dans le cas des parcours typiques (appelés encore parcours normés). Autrement dit, témoigner dans un texte permet en partie (et uniquement en partie!) l'attribution d'un niveau hiérarchique plus élevé12. L'existence du lien entre publication et promotion (même s'il reste relatif) est confirmée par les résultats de l'analyse longitudinale qui met en lumière des changements dans le nombre des publications proposées par chaque cadre

11 Taylor (1993) souligne que les mots « auteur » et « autorité » possèdent la même racine latine (auctor) qui suggère qu'étudier l'autorité, c'est révéler qui agit sur le texte ou qui autorise sa publication dans un moment spécifique, mais aussi comment les textes attribuent l'autorité aux humains et à l'organisation.

12 Pour un membre du personnel pratiquant un métier atypique. 
de direction qui varie dans le temps : avant, pendant et après l'attribution de la promotion. Ces changements indiquent que certains cadres de direction proposent davantage de publications avant et pendant la promotion. C'est grâce à l'analyse longitudinale et quantitative que le phénomène d'autopromotion est mis en lumière. Il consiste à obtenir des promotions non seulement pour le coauteur, mais aussi pour l'auteur des textes (celui qui propose, en l'occurrence, le cadre de direction). Toutefois, ce résultat nécessite d'être relativisé : le lien entre publication et attribution de promotion n'est qu'un des éléments (parmi beaucoup d'autres) qui expliquent la participation des cadres de direction et des personnels dans l'élaboration de Passerelle. Ces résultats obtenus n'épuisent donc pas toutes les raisons pouvant expliquer leur participation et leur prise de choix rédactionnel.

Mais les objectifs des cadres de direction, lorsqu'ils proposent des publications, ne sont certainement pas identiques, de même que toutes les publications proposées par eux ne sont pas suivies de promotions. Les résultats d'analyse précisent que quand les publications ne sont pas suivies de promotions, il s'agit de textes contenant des témoignages des personnels qui exercent des métiers typiques de l'organisation (49 textes). La possibilité d'obtenir une promotion pour ces personnes est faible à cause de leurs carrières à parcours normé. Dans ce cas, les cadres de direction proposent et autorisent les témoignages non pas pour obtenir des promotions, mais pour octroyer un certain type de reconnaissance, une valorisation qui est appelée ici thymos. Si le thymos est employé dans cette recherche, c'est parce qu'il y a une nécessité de contourner le sens commun et d'esquisser non pas une quelconque valorisation personnelle ou professionnelle, mais le processus d'attribution de cette valorisation entre un auteur et un coauteur à travers les textes organisationnels. Cette notion exprime l'existence d'un phénomène discursif qui émerge entre deux ou plusieurs personnes de positionnements hiérarchiques inégaux. Ce phénomène d'attribution des promotions et d'octroi du thymos se caractérise souvent par un décalage des publications dans le temps : les cadres de direction temporisent les publications des textes déjà proposés et rédigés ou décalent une seconde publication portant sur un sujet déjà publié précédemment. Ce décalage, appelé " marge d'action communicationnelle ", une notion qui mérite des approfondissements futurs pour devenir un concept, est très fréquent et vise à « avoir du marbre $»^{13}$, à garder sous le coude des textes tout prêts et de les publier dans un temps voulu, choisi. Cette stratégie de temporisation textuelle consiste à utiliser le sujet au bon moment, dans un contexte perçu comme favorable pour atteindre certains objectifs spécifiques, comme l'attribution des promotions et l'octroi du thymos.

13 «Marbre » désigne la table de marbre poli, puis table métallique, sur laquelle sont montées les pages d'un journal ou d'un livre avant leur impression. Ces tables ont disparu aujourd'hui, mais le terme est resté et désigne (en presse écrite) les articles en réserve, rédigés, non publiés, restés sur le marbre. 


\section{De quoi parlent les textes du journal interne ?}

Ce décalage n'est pas seulement une temporisation des étapes d'élaboration des publications, mais aussi du contenu des textes qui est constitué autour des différents événements organisationnels suivant le fil rouge de l'actualité. Les résultats d'analyse montrent que Passerelle parle de trois types d'événements : construits autour d'une actualité régionale, nationale, ou nationale et régionale à la fois. Les textes qui parlent des événements constitués autour d'une actualité nationale (16 cas) sont appelés événements pour les distinguer dans un premier temps des deux autres types. Ils sont proposés et publiés par obligation car institutionnels et politiques. À travers les événements, le journal interne se transforme en miroir des actualités nationales émergentes à l'extérieur de l'organisation qui n'exigent pas de transformations (de fonctionnement, de structure ou d'organigramme) internes au sein de l'organisation étudiée.

Les textes qui portent sur des événements constitués autour d'une actualité nationale et régionale à la fois $(29$ cas $)$ sont différents. À leur origine, il y a toujours une actualité nationale, mais ils exigent des transformations concrètes à l'intérieur de l'organisation (adoption de nouvelles missions, restructuration d'une unité, changement de l'organigramme officiel, etc.). Ces actualités sont localisées (de l'extérieur vers l'intérieur de l'organisation) via les textes $\mathrm{du}$ journal interne et se transforment en événements signifiants. Ce type d'événements est appelé, à l'instar de Philippe Zarifian (1995), « provénement qui est, dans la vie organisationnelle, quelque chose qui fait événement, et en même temps est construit en tant qu'événement. Ce sont des événements provoqués, localisés dans l'organisation grâce aux textes du journal interne à partir d'un environnement institutionnel extérieur.

Par ailleurs, la majorité des textes étudiés (100) parlent d'événements constitués autour des actualités régionales : leur origine interne ne peut pas être associée à un fait observé extérieur (national). Pour les distinguer des événements et des provénements, nous proposons de les appeler " artévénements " (en référence à un événement artificiel) : un événement construit par et dans l'organisation qui se globalise et devient présent grâce à sa publication dans le journal interne. La construction des « artévénements » se réalise à travers l'acte de proposition des publications par les cadres de direction : aucune actualité objective ne permet d'expliquer leur existence.

Bien que la majorité des textes de Passerelle parle des « artévénements », les trois types de textes ont une capacité d'agir : ils sont suivis soit de promotion/ autopromotion, soit du thymos. Mais si Passerelle parle majoritairement des événements artificiels, non existants dans un environnement extérieur et objectif, quelle est leur importance et que changent-ils en pratique?

\section{Qu'est-ce que le journal interne fait et fait faire à l'organisation qui le produit ?}

Depuis son lancement, des missions stratégiques officielles reposent sur le journal interne. Créé dans le cadre du projet d'entreprise du directeur, il 
doit parler du décloisonnement des unités et des projets interservices. Nos résultats montrent que ces missions officielles sont finalement peu articulées par les différents textes : ils ne portent ni sur le projet d'entreprise ni sur des projets transversaux. L'association impossible du projet d'entreprise et de Passerelle montre qu'ils ont des temporalités déconnectées. Si le projet prévoit une action dans un temps long à venir, le journal interne agit dans le moment présent. L'incapacité d'associer les deux temporalités est une des causes probables de cette impossible association, sans pour autant l'épuiser. Une enquête de lectorat sur le journal interne ${ }^{14}$ montre que la majorité des personnels ne connaît pas le projet d'entreprise, Passerelle ne l'évoque d'ailleurs que très peu. Les transformations stratégiques, visées lors du lancement du journal interne ne se traduisent pas dans la réalité : elles restent méconnues pour la majorité des membres du personnel.

Si Passerelle parle peu du projet d'entreprise, elle dit en revanche beaucoup sur le service Communication chargé de sa mise en place et coordination. Ce service, tardivement créé au sein de l'organisation étudiée, est atypique et à la marge des métiers les plus pratiqués. Peu valorisés et reconnus, les communicants et leurs réalisations sont en manque de légitimité. D'autres contraintes institutionnelles pèsent sur ce service : il est visé par un plan de réduction de l'effectif salarié et par un projet de mutations internes, voire de morcellement du service. À travers les textes métacommunicants, qui mettent en valeur le travail des communicants, le responsable du service Communication rend davantage visibles les compétences des membres de son équipe et leur savoir-faire. La légitimité du service est ainsi renforcée, le métier de communicant valorisé et l'intégrité du service préservée. Si la création du journal interne est officiellement prévue dans le cadre du projet d'entreprise pour porter des objectifs concrets, il n'accomplit pas les missions officielles qui lui sont attribuées. Sa capacité d'agir- son agentivité - est donc initialement imprévue et inattendue. Elle est construite collectivement dans un contexte spécifique institutionnel, mais irrigué par de multiples possibilités d'agir.

\section{Conclusion}

Nous avons montré à travers cette recherche que le journal interne, moyen principal de la communication interne, est un objet de recherche complexe qui est pertinent pour comprendre le rôle organisant de la communication au sein des organisations. Appréhendé comme le résultat d'une action collective partagée entre plusieurs individus actifs, son processus d'élaboration révèle comment les multiples auteurs et coauteurs plus ou moins autoritaires détournent, "bricolent » et instrumentalisent le journal interne pour atteindre des finalités personnelles. Il est donc un objet qui permet de concevoir les organisations comme un mode d'existence collectif

14 Elle est lancée par le service Communication un an après la création de Passerelle. Elle n'est pas scientifique et doit être prise avec beaucoup de recul. 
(Latour, 2012) particulier : animé par des individus actifs qui poursuivent des finalités spécifiques et organisé spatialement et temporairement par de multiples processus communicationnels à travers les interactions qui rendent possible l'actions collective.

Bien que cette recherche soit une étude de cas isolé d'un journal interne, elle peut donner lieu à des travaux à venir. Le journal interne, un objet de recherche " abandonné » par les sciences de l'information et de la communication, est ici " revisité " à travers la communication organisationnelle et les théories des organisations. Son élaboration collective permet de mieux comprendre l'articulation entre les objectifs des individus et les enjeux collectifs au travail qui sont souvent très divergents, des perspectives qui permettent de faire avancer les connaissances sur l'intelligence collective, une question à laquelle les chercheurs en communication s’intéressent ces dernières années.

\section{BIBLIOGRAPHIE}

AGNÈS Y., DURIER M., L'entreprise sous presse, Paris, Dunod, 1992.

ALDEBERT B., ROUZIES A., «L'utilisation des méthodes mixtes dans la recherche française en stratégie : constats et pistes d'amélioration ", 20ème colloque de l'AIMS, 6-9 juin 2011, Nantes.

ALMEIDA N., Les promesses de la communication, Paris, PUF, 2011.

APPEL V., « 1970 - 1995 : 25 ans de presse d'entreprise en France », Communication et langages, 114,1997, p. 28-35.

ARGENTI P., "How Technology Has Influenced the Field of Corporate Communication ", Journal of Business and Technical Communication, 20, 3, 2006, ABI/ INFORM Global, p. 357-370.

AUSTIN J., Quand dire, c'est faire, Paris, Éditions du Seuil, 1970.

BOTH A., " Le journal interne d'entreprise, Ethnographie d'une mission impossible ? ", Ethnologie française, 1-36, 2006, p. 45-54.

BOURCIER D., VAN ANDEL P. dir., « La sérendipité. Le Hasard heureux », Actes du colloque La sérendipité dans les sciences, les arts et la décision », Cerisy-La-Salle, 20 et 30 juillet 2009, Paris, Hermann Éditeurs, 2011.

BOUZON A., MEYER V. coord., La communication organisationnelle en question, Paris, L'Harmattan, 2006.

CARAYOL V., "Limpossible audit de communication interne ", Communication \& organisation [En ligne], 5, 1994, mis en ligne le 26 mars 2012, consulté le 8 mars 2013. URL : http://communicationorganisation.revues.org/1701.

COOREN F., "Textual agency: How texts do things in organizational settings ", Organization, 11/3, 2004, p. 373-393.

COOREN F., Manière de faire parler. Interaction et ventriloquie, Paris, Le bord de l'eau, 2013.

DÉCAUDIN J.-M., IGALENS J., La communication interne, Paris, Dunod, 2006. 
DEETZ S., "Theoretical and Methodological Issues », in JABLIN F., PUTNAL L. eds., The New Handbook of Organizational Communication, SAGE Publications, 2005, p. 3-39.

DELEY N., "Qui parle dans le journal d'entreprise ? Etude de cas », Études de Communication, 16, 1995, p. 109-132.

DENIS J,. « Les nouveaux visages de la performativité », Etudes de communication, 29, 2006, p. 8-24.

DÉTRIE P., BROYEZ C., La communication interne au service du management, Paris, Editions Liaisons, 2003.

FAIRHURST G., PUTNAM L., "Organizations as Discoursive Construction ", Communication Theory, Fourteen: One, 2004, p. 2-26.

GIORDANO Y., MUSCA G., « Les alpinistes dans l'imprévu. Pour une approche naturaliste de la décision ? ", Revue française de gestion, 225/6, 2012, p. 83-107.

GIROUX N., " La communication interne : une définition en évolution ", Communication Eo organisation, [en ligne], 5, 1994, mis en ligne le 26 mars 2012, consulté le 20 janvier 2013.

HONNETH A., La lutte pour la reconnaissance, Paris, Le Cerf, 2000.

HONNETH A., "Reconnaissance et reproduction sociale ", in PAYET J.P., BATTEGAY A. éd., La reconnaissance à l'épreuve, Lille, Presses Universitaires Septentrion, 2008, p. 45-58.

LABASSE P., «L'événement dans la politique de communication interne », Les cabiers de la communication interne, 19, 2006, p. 20-23.

LAMBERT J., La presse d'entreprise, Paris, PUF, 1993.

LARDELLIER P., "Paradoxes et devenirs de la presse d'entreprise française ", Communication et organisation, [En ligne], 13, 1998, mis en ligne le 26 mars 2012, consulté le 29 mars 2013. URL : http:// communicationorganisation.revues.org/2046.

LATOUR B., «Une sociologie sans objet ? Remarques sur l'interobjectivité », Sociologie du travail, 4, 1994.

LATOUR B., Enquête sur les modes d'existence: une anthropologie des modernes, Paris, Editions La Découverte, 2012.

MEAD G. H., Mind, self and society, Chicago, Chicago University Press, 1934, trad. française, L'esprit, le soi et la société, Paris, PUF, 1993.

MORILLON L., "Dans un contexte de fusion interculturelle, quelle évolution des marqueurs de culture dans le journal de communication interne ? ", in Colloque international "Culture des organisations DISTIC », Nice 2005.

MORILLON L., " Le journal de communication interne, un agent des enjeux stratégiques organisationnels », Semen, 28, 2009, p. 31-51.

MORIN E., «Le retour de l'événement », Communications, 18, 1972, p. 6-20.

MUMBY D., STOHL C., " Disciplining organizational communication studies », Management Communication Quarterly, 1996, McQ 10, 1, ABI/INFORM Global, p. 50-72.

NICOTRI V., « La communication interne comme récit : de l'intériorisation à la convention », Communication et langages, 130, 2001, p. 100-118. 
SANDRI E., «La sérendipité sur internet : égarement documentaire ou recherche créatrice ? ", Cygne noir, 2013, [En ligne] :<http://www.revuecygnenoir.org/numero/ article/la-serendipite-sur-internet> (consulté le 25 mars 2013).

SEARLE J., Les actes de langage, Paris, Hermann, 1972.

STRAUSS A., La trame de la négociation. Sociologie qualitative et interactionnisme, Paris, L'Harmattan, 1992.

TAYLOR J., Rethinking the Theory of Organizational Communication, How to Read an Organization, Norwood, New Jersey, Ablex Publishing, 1993.

TAYLOR J., VAN EVERY E., The Emergent Organization. Communication as Site and Surface, Mahwah, NJ, Lawrence Erlbaum Associates, 2000.

ZARIFIAN P., Le travail et l'événement, Paris, L'Harmattan, 1995.

Résumé : Cet article propose d'étudier le journal interne en train de se faire et se focalise sur le processus de son élaboration quotidienne. Quelles sont les stratégies personnelles de ceux qui contribuent à l'élaboration du journal interne ? Pourquoi s'y investissent-ils et qu'y écrivent-ils ? Pour répondre à ces questionnements, nous effectuons une observation participante au sein du service Communication d'une organisation qui met en place un journal interne dans le cadre du projet d'entreprise. Après une période de deux ans d'observation, nous procédons à une analyse mixte et longitudinale de 11 numéros du journal interne (145 textes) depuis sa création. Nos résultats permettent de démontrer comment le journal interne est instrumentalisé et utilisé par une multitude d'auteurs pour atteindre des finalités personnelles, mais aussi pour agir collectivement.

Mots-clés : journal interne, communication interne, communication organisationnelle.

Abstract : This research aims to better understand how organizational communication texts contribute to the setting of a strategic discourse and a textual agency by focusing on the process of elaboration of an in-house journal. Our main topics of interest are the personal strategies of those who contribute to the preparation of the in-house journal, the reasons for their day-today involvement and the change it brings to the working practice. Our approach is structured around participant observation within the Communication department of Préventika. Based on the data collected for a period of two years, we conduct a mixed methods analysis (thematic and longitudinal) of eleven editions of the in-house journal since its creation. This research challenges the classical theories which account for strategic missions of the in-house journal and emphasize the role of the texts as strategic organizational individual and collective action.

Keywords : In house journal, Internal comunication, Organzational communication. 